\title{
MANAGEMENT
}

\section{Leiten, steuern, führen}

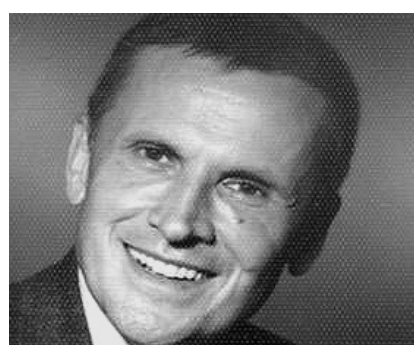

VON FRIEDHART HEGNER

Dr. Friedhart Hegner (67) ist Geschäftsführer der ISMV Dr. Hegner \& Partner $\mathrm{GmbH}$ (Institut für Sozialplanung, Management und Verwaltung) mit Sitz in Berlin und Bielefeld. Nach mehrjähriger Praxisarbeit ist er seit drei Jahrzehnten in der Organisationsforschung und Organisationsberatung tätig.

Internet http://www.ismv.de
In Umbruchzeiten sind Führungskräfte besonders gefordert: Leiten, Steuern, Führen sind die zentralen Aufgaben für das Management. Entscheidend dabei sind klare und gut abgestimmte Wertorientierungen des Unternehmens kombiniert mit einem transparenten Zielsystem.

Die angesichts der Staatsverschuldung absehbaren Engpässe in den öffentlichen Haushalten veranlassen viele Unternehmen aus dem Sozial- und Gesundheitsbereich dazu, sich vorbeugend wieder stärker auf zwei Grundsätze zielbezogenen Managens zu besinnen:

- Das Qualitätsniveau und die Innovationskraft im Wettbewerb müssen selbst bei scharfem Preisdruck gesichert werden.

- Die Verschwendung, der unnötige Verschleiß und die dafür meist ursächlichen Methodenfehler müssen nachdrücklich bekämpft werden.

Beides erfordert ein hohes Engagement und große Umsicht der Mitarbeitenden. Was sich dabei immer wieder bewährt hat, lässt sich in die drei Stichworte leiten, steuern und führen zusammenfassen. Der nachfolgende Text basiert dabei auf Erfahrungen unseres Teams bei der Organisationsberatung in mehr als 300 Unternehmen aus Industrie und Dienstleistungsbereich (zwischen 50 und 15.000 Mitarbeitern) in den Jahren seit 1975, darunter knapp 100 Einrichtungen und Träger aus der Sozial- und Gesundheitswirtschaft. Dabei war und ist die vergleichende Auswertung von Fachliteratur ein ständiger Begleiter.

\section{Leiten als Organisationsmittel}

Zentrale Bedeutung für wirksames Koordinieren der Handlungen dutzender oder hunderter Mitarbeiterinnen und Mitarbeiter haben klar erkennbare sowie gut abgestimmte Wertorientierungen des Unternehmens kombiniert mit einem transparenten Zielsystem. Das zu erarbeiten oder zumindest auf den Weg zu bringen, gehört zu den Kernaufgaben jeder Leitungskraft. Es ist Teil des Prozesses der Zielfindung und Zielsetzung als Vorstufe der operativen Zielrealisierung.

Die Struktur, die innere Ordnung des $\mathrm{zu}$ entwickelnden Zielsystems entfaltet dann die stärkste Zugkraft, wenn sie stabil und variabel zugleich ist. Sie muss gerade in Zeiten des Umbruchs dazu taugen

- sowohl strategische Ziele darzustellen, die das Handeln, also das Denken, Fühlen und Verhalten (Sprechen, Tun usw.) über mehrjährige Zeitspannen ausrichten

- als auch operative Ziele abzubilden, die den Handlungsketten mehrerer Mitarbeiter und Leitungskräfte über Wochen oder Monate hinweg eine Orientierung und Ausrichtung geben, die sich im Folgezeitraum schon wieder verändern kann.

Mit den Worten »darstellen « und »abbilden « wird zweierlei angesprochen:

- Auf der Sachebene kommt es darauf an, die Kernelemente des unternehmensweiten Zielsystems so variabel zu gestalten, dass sie in unterschiedlichen Organisationsbereichen und Organisationsebenen sowie bei wechselnden Umfeldanforderungen handlungsleitend wirken.

- Auf der Kommunikationsebene geht es darum, das Zielsystem sprachlich und bildlich in einer allgemein verständlichen Form darzustellen, die trotz inhaltlicher Zielverschiebungen weitgehend stabil bleibt und gut im Gedächtnis haftet.

Tipp: Je nach den Umfeldanforderungen und gemäß den Besonderheiten der einzelnen Geschäftsfelder und Organisationseinheiten kommt es darauf an, Prioritäten zu setzen! Zu entscheiden ist, ob in einem Zeitraum - kurzfristig für Wochen und Monate, mittelfristig für Halbjabre oder Jabre, längerfristig für fünf bis 15 Jabre - die Uhr primär für Kapitalgeber und Ertragskraft oder für Kunden und Wettbewerbsstärke oder für Arbeitskräfte und Arbeitsbedingungen schlägt.

Basiswerkzeug des operativ-alltäglichen Leitens ist die jeweils situationsge- 
recht zu wählende Methode, also gemäß griechischem Wortstamm: die Art und Weise, wie ein Weg zum Ziel oder zur Lösung eines Problems beschritten wird. Wer sich darauf konzentriert, erkennt: Qualitätsmängel, Verschwendung und unnötiger Verschleiß sind in der Regel weniger durch fehlende Motivation und Konzentration der Mitarbeiter als durch Methodenfehler bedingt. Mit dem Begriff Methodenfehler werden hier bezeichnet: alle Arten von lückenhaften, unklaren, unstimmigen oder gar widersprüchlichen Regelungen des Procedere. Regelungen umfassen sowohl fachlich-instrumentelle Standards (z. B. technologischer, juristischer oder medizinischer Art) als auch sozial-integrierende Normen für die Art des
Tipp: In der Regel werden die Ziele den Mitarbeitenden entweder direkt durch dafür verantwortliche Leitungskräfte vorgegeben oder aber auf einem zweckmäßigen Umweg in Kraft gesetzt: Beispielsweise werden sie im Falle des Qualitätsmanagements oder des Controllings durch Fachkräfte ausgearbeitet und durch Weisungsbefugnisse, die ibnen von Leitungskräften übertragen wurden, verbindlich und handlungswirksam gemacht. Oder: Bei einer Zielvereinbarung zwischen Leitungskräften und Mitarbeitenden erhalten die verabredeten Standards oder Normen ibre Wirksamkeit durch die Einwilligung der weisungsbefugten Leitungskraft - und der Mitarbeitenden.

\section{"Qualitätsmängel, Verschwendung und Verschleiß sind in der Regel nicht Fehler der Mitarbeitenden, sondern falsche} Regelungen der Prozesse"

Kommunizierens und der Konfliktbewältigung bei der Arbeit.

Fachliche Standards und soziale Normen werden in einer jeweiligen Zeitspanne von dafür verantwortlichen Fach- und Leitungskräften erstens ausgearbeitet, zweitens durch Anweisung in Kraft gesetzt und damit drittens den Mitarbeitenden verbindlich vorgegeben.

Durch Standards und Normen wird festgelegt, wer auf welche Weise - also wie, mit wem und bis wann - bestimmte Arbeitsschritte oder ganze Aufgabenbündel zu erledigen hat. Sie werden zielbezogen und nicht als Selbstzweck geschaffen und gepflegt. Andernfalls sind sie ein Indiz für Bürokratismus und Ritualismus. Standards und Normen als organisatorische Hilfsmittel geben dem Handeln einzelner sowie dem Zusammenarbeiten mehrerer von zwei bis viele tausend - Mitarbeitender eine zweifache Richtschnur:

- Als Ergebnisstandards und Ergebnisnormen bilden sie zum Ersten einen verbindlichen Zielrahmen, also nicht bloß vage Visionen oder verworrene Zielsammelsurien

n zum Zweiten wirken sie - bildlich - als Wegweiser, Leitplanken oder Korridore in Form von Prozessstandards oder Prozessnormen. Sie bilden also fachlich begründete sowie durch Verhaltenserwartungen mit Nachdruck versehene Korridore für zweckmäßiges Handeln im Kontext zielbezogener Aktionsprogramme.

\section{Steuern}

Es gehört zum unverzichtbaren Werkzeug der konsequenten Zielverfolgung, situationsgerecht einzugreifen, wenn das Verhalten der Mitarbeitenden zum einen von den Prozessstandards und Prozessnormen abweicht, also die Korridorgrenzen überschreitet, oder zum anderen den vorgegebenen Grad der Zielerreichung verfehlt. Dann sind steuernde Maßnahmen der Zielrealisierung in Ergänzung zu denjenigen der Zielfindung und Zielsetzung unerlässlich. Zur Arbeit des Steuerns gehört das Justieren des Leitens mit zwei Komponenten:

- das eventuell nötige Nachbessern bei Zielen sowie damit verbundenen Standards und Normen für zu ergreifende oder zu unterlassende Maßnahmen, wenn sich die tatsächlichen Umfeldbedingungen oder die diesbezüglichen Erkenntnisse deutlich verändern, sowie

- das Justieren des Arbeitsverhaltens einzelner oder vieler, wenn es von den Standards oder Normen abweicht egal ob versehentlich oder gewollt, ob in guter oder böswilliger Absicht.

Ebenso wie die Fach- und Leitungsarbeit bei der Zielsetzung startet auch die steuernde Zielrealisierung zunächst auf der Sachebene. Das Steuern erfordert instrumentales Können bezüglich fachlicher Standards und sozialer Normen (z. B. Techniken der zielorientierten Gesprächsführung). Mit Hilfe geeigneter Methoden (z. B. Werkzeuge zur systematischen Lückenanalyse) ist viererlei zu prüfen:

- Sind die gesetzten oder vereinbarten Ziele ausreichend deutlich formuliert und unter den gegebenen Bedingungen erreichbar?

- Erweisen sich die Leitplanken der Wege zum Ziel, also die Standards und Normen für zu ergreifende Maßnahmen, als geeignet?

- Sind die bereitgestellten Sach- und Personalressourcen zweckmäßig und ausreichend?

- Was genau an dem abweichenden Arbeitsverhalten einzelner oder vieler trägt zur Zielverfehlung oder Korridorverletzung bei und muss deshalb verbessert werden?

Nach der Beantwortung dieser Fragen wird entschieden, was auf welche Weise zu justieren ist.

Diese vierfache Sachverhaltsklärung und Sachverhaltsentscheidung hat neben den sachlichen Vorteilen des Justierens einen positiven psychosozialen Nebeneffekt. Dabei geht es um die psychisch entlastende Wirkung der Aussicht, eventuell fehlerhafte Entscheidungen steuernd nachbessern zu können. Sie ist für diejenigen Leitungskräfte besonders wichtig, die sich generell sehr schwer tun, Entscheidungen zu treffen. Die Entscheidungsscheu oder gar Entscheidungsangst resultiert primär aus dem unangenehmen Gefühl, zwischen mehreren Zielen und Wegen wählen zu müssen, obwohl die entscheidungsrelevanten $\mathrm{Da}$ ten lückenhaft oder ambivalent sind und sich die Reaktionen der Entscheidungsbetroffenen nur teilweise vorhersehen lassen.

Tipp: Das oft allzu lange Zögern vieler Leitungskräfte angesichts ungewisser Entscheidungsfolgen lässt sich beim Training und Coaching für (angehende) Manager dadurch verringern, dass man die Option des späteren Steuerns und Justierens erläutert. Diese Option verringert den als belastend empfundenen Entscheidungsdruck in der Zielauswahlphase.

\section{Führungsarbeit}

Folgendes verdeutlicht den Zusammenhang zwischen Führen einerseits sowie Leiten und Steuern andererseits:

- Durch gut strukturierte Informationsarbeit wird bewirkt, dass die Mitarbeitenden die Standards und Normen sowie die angestrebten Ziele richtig verstehen und gedanklich nachvollziehen. Das sind die Mindestbedingungen für eine wirkungsvolle Leitungs- und Zielsetzungsarbeit. Im günstigsten Fall kann es durch gute Informationsarbeit sogar gelingen, dass die Beteiligten bezüglich der Ziele und Wegmarken die 
gleichen positiven Gefühle hegen wie die Leitungskraft - eventuell sogar mit Begeisterung.

- Je nach Art und Intensität der inneren Widerstände gegen die Umsetzung der Informationen in Alltagshandeln ist Überzeugungsarbeit nötig. Sie erfolgt in Form von wiederholter und hinsichtlich der Medien (Sprache, Schrift, Bild usw.) variabler Kommunikationsarbeit. Sie beleuchtet den jeweiligen Sachverhalt aus verschiedenen Blickwinkeln und Interessenstandpunkten. So lässt sich die Akzeptanz der Ziele, Standards und Normen aufseiten der Mitarbeitenden verbessern.

- Wenn das Verhalten der Mitarbeitenden zum einen von den Standards und Normen abweicht, also die Korridorgrenzen überschreitet, oder zum Zweiten den vorgegebenen Grad der Zielerreichung verfehlt, sind zunächst verständliche Informationen nötig, beispielsweise unmissverständliche sachliche Kritik an den abweichenden Aspekten des Verhaltens statt pauschaler Verurteilung. Sie sind eventuell zu ergänzen durch kommunikativen Austausch, beispielsweise Verständigung über den genauen Inhalt von verletzten Normen sowie auf Korrekturmaßnahmen. Im Wiederholungsfall hilft oft nur nachdrückliche Information mit Sanktionsandrohung. Durch derartige gut zu dosierende Handlungen auf der Kommunikationsebene sollen die steuernden Eingriffe auf der Sachebene (z. B. Anwendung von Sanktionen, oder aber Bereitstellung zusätzlicher Ressourcen) so wirkungsvoll wie möglich gemacht werden.

Bei der Wahrnehmung der genannten Aufgaben entwickeln Leitungskräfte im Laufe der Zeit einen mehr oder weniger markant ausgeprägten Führungsstil. Dieses Ensemble von persönlichen Mustern des Fühlens und Denkens prägt die Muster des Verhaltens gegenüber den Mitarbeitenden. Dabei geht es jedoch keineswegs nur um den Stil des Kommunizierens, sondern um den Stil des Kombinierens von Leiten, Steuern und Führen in Form eines mehrdimensionalen Netzes oder »Gitters «:

In einer Hinsicht wird der Stil des Handelns davon geprägt, wie stark eine Leitungskraft primär auf die Sachebene des Leitens und Steuerns fixiert ist und die Ebene des Kommunizierens mit anderen Akteuren untergewichtet. Unter einem zweiten Blickwinkel erkennt man den Führungsstil daran, auf welche Weise eine Leitungskraft in das Handeln anderer Akteure interveniert: eher dirigistisch, indem persönliche Entschlüsse ohne Eingehen auf Widerspruch mitgeteilt und Entscheidun- gen primär per Anweisung durchgesetzt werden? Oder eher konsensorientiert, indem vor dem persönlichen Entschluss zur Zielformulierung die Meinungen anderer abgewogen und zumindest beim justierenden Steuern berücksichtigt werden?

Tipp: Es gibt keinen einzig richtigen Führungsstil. Bewährt haben sich jedoch zwei Grundregeln:

- Den eigenen Stil der Aufgabenwahrnehmung - beispielsweise durch Rücksprache mit Mitarbeitenden - daraufhin prüfen, ob man primär als sachorientierte Leitungskraft oder vorrangig als konsensorientierte Führungskraft wahrgenommen wird.

- Von beidem profitieren, also einseitige Überzeichnungen in Richtung bloß sachorientiertes Leiten und Steuern ebenso vermeiden wie in Richtung ständiges Kommunizieren zwecks Konsensgewinnung - noch schlimmer: mit Rückzug auf völlig verschwommene Ziele und unter Verzicht auf justierendes Steuern.

Durch das situationsgerechte Kombinieren von Leiten, Steuern und Führen entsteht jenes Maß an Überzeugungskraft, das zum nachhaltig wirkungsvollen Koordinieren der Handlungen von Mitarbeitenden befähigt. Auf diese Weise lassen sich Qualitätsmängel ebenso drastisch reduzieren wie unnötiger Verschleiß und Verschwendung.

\section{Stiftungswesen}

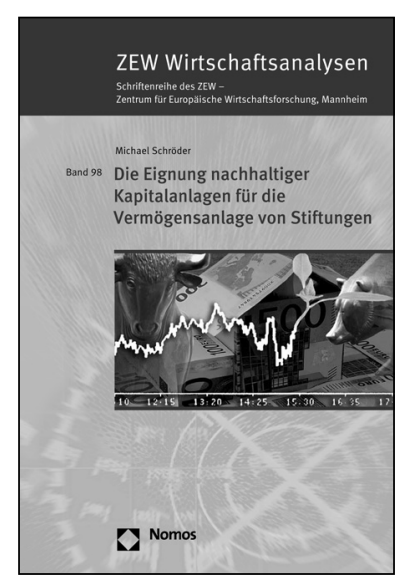

\section{Die Eignung nachhaltiger Kapitalanlagen für die Vermögensanlage von Stiftungen}

Von PD Dr. Michael Schröder

2010, 262 S., brosch., 59,- $€$, ISBN 978-3-8329-5662-2

(ZEW WirtschaftsanalysenSchriftenreihe des ZEW, Bd. 98)

Im Zentrum der Studie stehen die Untersuchung von Strategien der Vermögensanlage und ihre Bewertung aus der Perspektive gemeinnütziger Stiftungen. Von besonderer Bedeutung sind dabei nachhaltige Kapitalanlagen, die spezielle ethische, soziale und ökologische Auswahlkriterien anwenden. Sie werden einer umfassenden betriebs- und finanzwirtschaftlichen Analyse unterzogen.

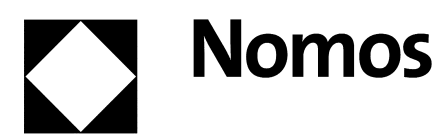

